\title{
An investigation of computer-assisted cognitive-behavior therapy in the treatment of depression
}

\author{
PAULETTE M. SELMI \\ Department of Psychology, Illinois Institute of Technology, Chicago, Illinois 60616, and \\ Department of Psychiatry, University of Wisconsin, Madison, Wisconsin 53706 \\ MARJORIE H. KLEIN and JOHN H. GREIST \\ Department of Psychiatry, University of Wisconsin, Madison, Wisconsin 53706 \\ and \\ JAMES H. JOHNSON and WILLIAM G. HARRIS \\ Department of Psychology, Illinois Institute of Technology, Chicago, Illinois 60616
}

\begin{abstract}
Increasing demands are being placed on a limited pool of mental health professionals to deliver a steadily growing array of effective treatments in an efficient and acceptable manner. The integration of behavioral technology and computing technology may provide a possible solution to the problem of resource limitations. An interactive computer-assisted program for the treatment of mild to moderate unipolar and dysthymic depressive disorders is described. The effectiveness of this treatment will be empirically examined.
\end{abstract}

In recent years, computing technology has been applied in a number of mental heal th areas: automated management information systems (Laska, Siegel, \& Bank, 1980), automated psychological testing (Fowler, 1980 ), prompting of physical and mental status examinations (Williams, Johnson, \& Bliss, 1975), behavior modification research and psychological assessment (Lang, 1980), computer-based interviews for gathering patient information (Greist \& Klein, 1980), and on-line psychiatric diagnosis (Erdman, Greist, Klein, Jefferson, Olson, \& Salinger, 1980; Erdman, Greist, Klein, Jefferson, Salinger, \& Olson, 1981; Greist, Klein, \& Erdman, 1976).

Despite the wide range of uses of computer assistance in mental health care and delivery, resistance to their implementation has been a major drawback. This has been especially true as the use of a computer is directed at the more "professional" aspects of mental health care (Lanyon, 1971), such as diagnosis, testing, and treatment. Resistance to change, however, is an expected component of the change process requiring that careful attention be devoted to the important issues involved on both sides so that an adequate understanding can be achieved (Byrnes \& Johnson, 1981).

Lanyon and Johnson (1980) suggested that the controversy over the use of technology in mental health care

This article is based on a dissertation to be submitted in partial fulfillment of the requirements for the PhD degree in the Department of Psychology at the Illinois Institute of Technology. This research is being funded in part by the National Institute of Mental Health, Grant MH-16464-01. may be due to a misunderstanding of the nature of technology and its relation to the social and behavioral sciences. Technology is a term referring to procedures that are implemented to increase and improve productivity. This is accomplished by replacing highly skilled "craftsmen" with less skilled "workers" whose productivity is increased through the use of highly sophisticated tools, with an end result of reducing the cost of production. Given this definition, it can be said that mental health care is "pretechnological": A highly skilled craftsman (psychiatrist, psychologist, social worker, etc.) completes the entire work process (clinical interviewing, testing, therapy), producing less output at a higher cost per unit (Lanyon \& Johnson, 1980). Lanyon (1971) noted that areas such as mental health that lack technology will become more expensive. Thus, three courses of action are available to respond to the needs placed upon mental health delivery systems: (1) accept the increasingly greater expense for mental health services, (2) attempt the development of the appropriate technologies to respond to the mental health needs, or (3) experiment with radical changes in ways of meeting society's mental health needs.

It is unlikely that patient/consumers and third-party payers can afford or will accept increasing expenses. Mental health services, as we know them today, are affordable only to those who have the resources, either private or by third-party payment. Third-party payers, ultimately the public, have been demanding answers to questions regarding the quantity, quality, and cost of psychosocial treatments (Parloff, 1979), and mental 
health professionals are now being held more accountable for the treatments they provide (Schwitzgebel \& Schwitzgebel, 1980). Relative short-term, effective, and cost-efficient psychotherapies will be the obvious psychotherapeutic treatment of choice for most patients (Strupp, 1978).

The development of the appropriate technologies to respond to the demands for mental health care, Lanyon's (1971) second option, is being supported in new research on depression. This research includes the development of short-term psychotherapies (Beck, Rush, Shaw, \& Emery, 1979) and the use of behavioral technologies in the treatment of depression. The therapy being considered in this project, cognitive-behavior therapy, is relatively short-term and has been found to be effective in a circumscribed set of psychological disorders, especially in the treatment of depression (Beck et al., 1979).

A number of research developments in the past years have utilized computing in psychological treatment. These efforts have been accompanied by a great deal of resistance (Spero, 1978; Weizenbaum, 1976). Unfortunately, the resistors "are often blind to large, understaffed state hospitals and community mental health centers. which do little but triage patients, often for unavailable or inefficient the rapies" (Greist \& Klein, 1981 , p. 771). Colby (1967) has also stated that it is dehumanizing to "herd" thousands of patients into these hospitals, so that use of computers may provide a chance to rehumanize individuals who are being dehumanized by our psychiatric system.

Weizenbaum's (1966) computer program, ELIZA, was a crude simulation of Rogerian psychotherapy and was written primarily to demonstrate the effectiveness of the computer in certain text manipulations. ELIZA was never tested in a clinical setting, nor did its author believe in the ethical acceptability of such a pursuit (Weizenbaum, 1976). On the other hand, Colby and his associates have developed programs with the intent of providing patients psychotherapeutic experiences (Colby, Watt, \& Gilbert, 1966). Their programs allow subjects to communicate with the computer, free form, by typing anything they wish. While the intent of the programs is to help patients in the same way a psychotherapist does (i.e., by questioning, clarifying, reflecting feelings, rephrasing, and some times interpreting), almost everyone who has interacted with these programs has felt annoyed or frustrated because the computer often fails to answer pertinent questions (Colby et al., 1966).

Other efforts to develop programs have continued, but widespread interest in these methods has not been evident. The textual problems involved are complex, and the programs take years to develop. It is unlikely that computers will be used to deliver psychotherapy in the form that it is today, but computers may provide packaged, action-oriented, directed therapies for specific problems (Johnson, 1979). As research in psychotherapy continues toward the development of short-term approaches for target problems, computer-assisted versions will be easier to implement.

This has been the case in the behavior therapies. There have been many attempts to automate behavioral treatment (Lang, 1969), as described by Elwood (1975), but they generally have not involved the use of interactive systems between patient and computer. A system for the treatment of phobics (Ghosh, 1981) has recently been evaluated by using three treatment conditions: human therapist, computer therapist, and a group using a self-help instruction manual. The study has not been completed, but preliminary results indicate that the computer is yielding positive results. Patient acceptance of the computer has been positive (Carr \& Ghosh, in press). Other studies have also found that patients are willing to share sensitive information with a computer and some prefer it to talking to a clinician (Greist \& Klein, 1980; Greist, Klein, \& VanCura, 1973; Lucas, Mullin, Luna, \& McInroy, 1977).

Patient acceptance of computer-assisted interviewing is one major advantage in the continued efforts to develop treatment packages for computer delivery. Another advantage is that treatments can be made available to more patients at a substantial monetary savings (Elwood, 1975; Klingler, Miller, Johnson, \& Williams, 1977) and can be more convenient, because a computer can be available at any time of the day, any day of the week. There are also advantages from a clinician's point of view: Technicians can monitor the computer treatment, and a therapist's time is freed for more difficult cases. A research advantage is that greater experimental control can be achieved over interpersonal variables thought to be important in psychotherapy. For all of these reasons, additional research is needed to develop and test computerassisted treatments.

\section{COMPUTER-ASSISTED COGNITIVE BEHAVIOR THERAPY}

We have developed a computer-based program for the delivery of cognitive-behavior therapy. This method of treatment was chosen for computerization because of its apparent effectiveness (Beck, 1976; Beck et al., 1979; Kovacs, 1980; Kovacs, Rush, Beck, \& Hollon, 1981; Rush, Beck, Kovacs, \& Hollon, 1977; Shaw, 1977; Taylor \& Marshall, 1977) and because it is short-term, with relatively well-defined treatment strategies that are amenable to a computerized application.

Three principles are central to cognitive therapy: (1) All moods are created by cognitions or thoughts, (2) thoughts in depression are negative, and (3) negative thoughts are almost always distorted in some ways. The basic goal of cognitive-behavior therapy is to alleviate dysphoric affect in the depressed patient through the direct modification of dysfunctional thoughts and aber- 
rant thinking patterns. This goal is achieved through the use of various behavioral and cognitive therapeutic techniques, and hence the name, cognitive-behavior therapy. A treatment manual has been written that details a usual course in treatment (Beck et al., 1979).

Cognitive-behavior therapy usually involves 20 treat ment sessions over a 12 -week period. The computerassisted treatment package that has been developed involves six to eight sessions over a 6 -week period because the computerized techniques are experimental and "psychoeducational" in nature.

\section{Program Description}

The agenda for each of the computerized interviews follows the protocol for outcome studies prepared by Beck et al. (1979) as closely as the medium permits. The system permits only limited free text manipulations and does not have the capability to interpret large amounts of free form responses. Therefore, greater use is made of multiple-choice items, case vignettes, and generic forms of identifying and dealing with dysfunctional thoughts.

In Session 1, rapport is established. The computer program introduces itself as MORTON, "speaks" to the subject in the first person, and addresses the subject by his or her first name. Expectations about therapy and the major complaints are then elicited. This information is used as a branching point at the end of the session to determine an appropriate homework assignment. MORTON next administers the Beck Depression Inventory (BDI) (Beck, Ward, Mendelson, Mock, \& Erbaugh, 1961), which is used as a weekly indicator of the subject's level of depression. MORTON then explains in detail the cognitive theory of emotion on which the therapy is based and presents a series of multiple-choice questions to determine if the subject understands the model. Feedback is given for each answer the subject enters, and homework is assigned. This initial interview takes from 40 to $50 \mathrm{~min}$ to complete, as do the remaining interviews. Any materials needed for homework assignments are provided by the experimenter.

Session 2 is initiated with agenda setting and a repeat administration of the BDI. Feedback is given according to the fluctuation, if any, in the depression score from the previous session. The problems discussed in the first session are reviewed and redefined, if necessary. MORTON and the subject then review the homework assignment from the week before. Reactions, questions, and clarification of the assignment are completed next. MORTON then reviews the content of the last session concerning the principles of cognitivebehavior therapy, and again. the subject is presented multiple-choice items to check for mastery of the principles. Homework assignments are chosen, and reactions toward the interview are elicited. Figure 1 provides an example of an interaction in the second session.

In Session 3, an agenda is set, the BDI is admini- stered, and feedback on the BDI score is given. Homework assignments are reviewed. This is followed by a discussion of dysfunctional automatic thoughts. Several case examples are given to aid the subject in determining automatic thoughts that cause depression. Homework assignments consist of having the subject write down specific situations that precipitate feelings of depression.

The remaining sessions proceed in a similar fashion, with greater emphasis placed on identifying automatic thoughts, defining the cognitive distortions in these thoughts, and teaching the patient how to arrive at rational responses to the automatic thoughts. The subject is prepared for termination in the last session and is given guidance for dealing with future depressions.

\section{Program Operation}

The therapeutic interviews are written by means of Converse. a programming technique that uses MIIS, a dialect of MUMPS. Converse is an "interview driver" that makes it possible to create, edit, administer, and summarize computer-based interviews easily (Bloom, White, Beckley, \& Slack, 1978). The interviews are written and administered on cathode-ray tube terminals and run on a Data General Eclipse C-330 with $512 \mathrm{~KB}$ of core memory and $384 \mathrm{MB}$ of disk memory.

Converse allows free text responses, multiple-choice responses. numerical responses, and responses corresponding to tabled entries prepared by the interview writer. The subject enters answers into the system by means of a typewriter-like keyboard. Converse is particcularly suited for the cognitive-behavior therapy interviews because of its capacity to handle the complex sets of logic required and the relative ease with which extensive amounts of text can be entered.

\section{Program Evaluation}

The effectiveness of the computer-assisted cognitive. behavior therapy treatment package will be empirically examined in an outcome study utilizing three treatment groups: computer therapist, human therapist, and a treatment-on-demand control group. The human therapist will follow a treatment protocol similar to the computer therapist's protocol. The treatment-on-demand control group will be offered nondirective, supportive treatment by a human therapist, when necessary, up to a total of $2 \mathrm{~h}$ for the duration of the 6 -week period. This group will be offered the computer-assisted therapy if preliminary results indicate that the program has not been harmful.

Subjects will be volunteers responding to newspaper advertisements calling for people interested in studies in depression. They will be carefully screened with an extensive clinical interview, the Schedule for Affective Disorders and Schizophrenia (SADS) (Endicott \& Spitzer, 1978), and only those who meet the Research Diagnostic Criteria (Spitzer, Endicott, \& Robins, 1978) for a mild to moderate nonpsychotic unipolar depressive 
MORTON: Last week, we talked about the principles of Cognitive Therapy.

If you recall, Principle \#1 stated that feelings are caused by cognitions or thoughts.

Today, I want for us to examine this principle in greater detail.

How does this sound to you?

1. Sounds fine. Let's continue.

2. I would like a brief review of the major points you made last week, before continuing.

SUBJECT: 1

MORTON: Good. Let's try a few examples...

If a person were to think to him or herself "I am inferior"

what is likely to occur?

1. The person would compare himself to others and find faults in himself and feel bad.

2. Probably would feel real low about himself.

3. He would get angry.

(choose the best answer(s))

SUBJECT: $\quad 1,2$

MORTON: You're right John. By saying to himself, "I'm inferior", he would compare himself to others, be very critical of himself, and probably get depressed.
Let's try another ...

Suppose a boss gets upset and starts yelling at an employee. The employee thinks:

"I know it. I can't do anything right!"

How will the employee feel?

1. Anxious

2. Depressed/low

3. Angry

SUBJECT: 2

MORTON: Good work!

Now, suppose a boss starts yelling at the employee and the employee thinks:

"That guy has got the nerve! I won't stand for this!"

How will the employee feel?

1. Anxious

2. Depressed/low

3. Angry

SUBJECT: 2

MORTON: No, he wouldn't feel depressed, John. He would be angry, because the employer has put him down and the employee won't stand for that. Perhaps the employee has thought that the "put down" was unjustified.

Can you see from this example that different thoughts about the same situation can produce different feelings?

Figure 1. Example of an interaction in a therapy session.

disorder will be included in the investigation. Volunteers will be randomly assigned to one of the three conditions.

Pretest measurements will include the SADS interview (Endicott \& Spitzer, 1978), the Hamilton Depression Rating Scale (HRS-D), which will be rated from the SADS interview (Endicott, Cohen, Nee, Fleiss, \& Sarantakos, 1981), and the Hopkins Symptom Checklist (SCL-90) (Derogatis, Lipman, Rickels, Uhlenhuth, \& Covi, 1974). In addition, each subject will meet with a rater, blind to the treatment condition, prior to treatment, to determine goals for therapy, according to the goal-attainment scaling procedure outlined by Kiresuk and Sherman (1968). The BDI will be administered before treatment and during all sessions, but it will not be used as a major outcome variable. Posttreatment measurements will inciude a SADS-change version (SADS-C) interview (Endicott \& Spitzer, 1978), an HRS-D rated from the SADS-C, and the SCL-90. Each subject will also meet with a rater, blind to the treatment condition, to assess the progress made toward the goals specified prior to treatment. A 2-month followup assessment session using the posttreatment measures will be conducted. Within- and between-groups differences will again be statistically analyzed to de termine treatment effects.

\section{SUMMARY}

A computer-assisted packaged treatment program for depression has been described. This program will be evaluated by randomly assigning homogeneous samples of depressed individuals to one of three groups: computer therapist, human therapist, or treatment-ondemand control. Prettest and posttest measurements of depression and other indicators of psychopathology will be taken to determine the differential effectiveness of the treatment programs. We hope that the development of computer-assisted packaged therapies, such as MORTON, can play a role in reducing mental health manpower shortages and in providing mental health care at a cost that is affordable. 


\section{REFERENCES}

Beck, A. T. Cognitive therapy and the emotional disorders. New York: International Universities Press, 1976.

Beck, A. T., Rush, A. J., Shaw, B. F., \& Emery, G. Cognitive therapy of depression. New York: Guilford Press, 1979.

Beck, A. T., Ward, C. H., Mendelson, M., Mock, J. E., \& Erbaugh, J. K. An inventory for measuring depression. Archives of General Psychiatry, 1961, 4, 561-571.

Bloom, S., White, R., Beckley, R., \& Slack, W. Converse: A means to write, edit, administer, and summarize computerbased dialogue. Computers and Biomedical Research, 1978, $11,167-175$.

Byrnes, E., \& Johnson, J. H. Change technology and the implementation of automation in mental health care settings. Behavior Research Methods \& Instrumentation, 1981, 13, 575-580.

Carr, A. C., \& Ghosh, A. Response of phobic patients to direct computer assessment. British Journal of Psychiatry, in press.

Colby, K. M. Computer simulation of change in personal belief systems. Behavioral Science, 1967, 12, 248-253.

Colby, K. M., Watt, J. B., \& Gilbert, J. B. A computer method of psychotherapy. Preliminary communication. Journal of Nervous and Mental Disease, 1966, 142, 148-152.

Derogatis, L. R., Lipman, R. S., Rickels, K., Uhlenhuth, E. N., \& Covi, L. The Hopkins symptom checklist (HSCL). Behavioral Science, 1974, 19, 1-15.

Elwood, D. L. Automation methods. In F. H. Kanfer \& A. P. Goldstein (Eds.), Helping people change. New York: Pergamon, 1975.

Endicott, J. E., Cohen, J., Nee, J., Fleiss, J., \& Sarantakos, S. Hamilton depression rating scale: Extracted from regular and change versions of the Schedule for Affective Disorders and Schizophrenia. Archives of General Psychiatry, 1981, 38, 98-103.

EndicotT, J., \& Spitze R, R. L. A diagnostic interview: The schedule for affective disorders and schizophrenia. Archives of General Psychiatry, 1978, 35, 837-844.

Erdman, H., Greist, J., KLein, M., Jefferson, J., Olson, W., \& Salinger, R. Computer consultation for psychiatric diagnosis. In J. F. O'Neill (Ed.), The Fourth Annual Symposium on Computer Applications in Medical Care. New York: Institute of Electrical and Electronics Engineers, 1980.

Erdman, H., Greist, J., Klein, M., Jefferson, J., Salinger, R., \& Olson, W. Clinical usefulness of a computer program for psychiatric diagnoses. In B. Shriver, T. Walker, R. Grams, \& R. Sprague (Eds.), Proceedings of the Fourteenth Hawaii International Conference on System Sciences. North Hollywood, Calif: Western Periodicals, 1981.

Fowle R, R. D. The automated MMPI. In J. B. Sidowski, J. H. Johnson, \& T. A. Williams (Eds.), Technology in mental health care delivery systems. Norwood, N.J: Ablex, 1980.

Gнosh, A. Therapeutic interaction and outcome in phobia. Unpublished doctoral dissertation, Institute of Psychiatry, London, 1981.

GREIST, J. H., \& KLEIN, M. H. Computer programs for patients, clinicians, and researchers in psychiatry. In J. B. Sidowski, J. H. Johnson, \& T. A. Williams (Eds.), Technology in mental health care delivery systems. Norwood, N.J: Ablex, 1980.

GREIST, J. H., \& KLEIN, M. H. Computers in psychiatry. In S. Arieti \& K. Brodie (Eds.), American handbook of psychiatry (2nd ed.). New York: Basic Books, 1981.

Greist, J. H., Klein, M. H., \& Erdman, H. P. Routine online psychiatric diagnosis by computer. American Journal of Psychiatry, 1976, 133, 1405-1408.

Greist, J. H., Klein, M. H., \& VanCura, L. J. A computer interview for psychiatric patient target symptoms. Archives of General Psychiatry, 1973, 29, 247-253.

Johnson, J. H. Technology. In T. A. Williams \& J. H. Johnson
(Eds.), Mental health in the 21st century. Lexington, Mass: Heath, 1979.

Kiresuk, T. J., \& Sherman, R. E. Goal attainment scaling: A general method for evaluating comprehensive community mental health programs. Community Mental Health Journal, $1968,4,443-453$.

Klingler, D. E., Miller, D. A., Johnson, J. H., \& Williams, T. A. Process evaluation of an on-line computer-assisted unit for intake assessment on mental health patients. Behavior Research Methods \& Instrumentation, 1977, 9, 110-116.

Kovacs, $M$. The efficacy of cognitive and behavior therapies for depression. American Journal of Psychiatry, 1980, 137, 1495. 1501.

Kovacs, M., Rush, A. J., Beck, A. T., \& Hollon, S. D Depressed outpatients treated with cognitive therapy or pharmacotherapy: A one-year follow-up. Archives of General Psychiatry, 1981, 38, 33-39.

LANG, P. J. The on-line computer in behavior therapy. American Psychologist, 1969, 24, 236-239.

LANG, P. J. Behavioral treatment and bio-behavioral assessment. In J. B. Sidowski, J. H. Johnson, \& T. A. Williams (Eds.), Technology in mental health care delivery systems. Norwood, N.J: Ablex, 1980.

LANyon, R. I. Mental health technology. American Psychologist, 1971, 26, 1071-1076.

LANYON, R. I., \& Johnson, J. H. Technology in mental health: A conceptual overview. In J. B. Sidowski, J. H. Johnson, \& T. A. Williams (Eds.), Technology in mental health care delivery systems. Norwood, N.J: Ablex, 1980.

LASKA, E. M., SIEGEL, C., \& BANK, R. Management information systems in mental health. In J. B. Sidowski, J. H. Johnson, \& T. A. Williams (Eds.), Technology in mental health care delivery systems. Norwood, N.J: Ablex, 1980.

Lucas, R. W., Mullin, P. J., Luna, C. B. X., \& McInroy, D. C Psychiatrists and a computer as interrogators of patients with alcohol-related illnesses: A comparison. British Journal of Psy. chiatry, 1977, 131, 160-167.

Parloff, M. B. Can psychotherapy research guide the policymaker? A little knowledge may be a dangerous thing. American Psychologist, 1979, 34, 296-306.

Rush, A. J., Beck, A. T., Kovacs, M., \& Hollon, S. Cognitive therapy and psychotherapy in the treatment of depressed outpatients. Cognitive Therapy and Research, 1977, 1, 17-37.

Schwitzgebel, R. L., \& Schwitzgebel, R. K. Law and psychological practice. New York: Wiley, 1980.

Shaw, B. F. Comparison of cognitive therapy and behavior therapy in the treatment of depression. Journal of Clinical and Consulting Psychology, 1977, 45, S43-551.

Speno, M. H. Thoughts on computerized psychotherapy. Psychiatry, 1978, 41, 279-288.

Spitzer, R. L., EndicotT, J., \& Rosins, E. Research diagnostic criteria: Rationale and reliability. Archives of General Psychiatry, 1978, 35, 773-782.

Strupp, H. H. Psychotherapy, research and practice: An overview. In S. L. Garfield \& A. E. Bergin (Eds.), Handbook of psychotherapy and behavior change (2nd ed.). New York: Wiley, 1978.

TAYLOR, F. G., \& Marshall, W. L. Experimental analysis of a cognitive-behavioral therapy for depression. Cognitive Therapy and Research, 1977, 1, 59-72.

WEIZENBAUM, J. ELIZA-A computer program for the study of natural language communication between man and machine. Communication of the Association for Computer Machinery, $1966,9,36-45$

WeIZENBAUM, J. Computer power and human reason: From judgment to calculation. San Francisco: Freeman, 1976

Williams, T. A., Johnson, J. H., \& Bliss, E. L. A computerassisted psychiatric assessment unit. American Journal of Psy. chiatry, 1975, 132, 1074-1076. 